\title{
Pharmacist-led quality control circle in sustained reduction of carbapenem-resistance at a Chinese tertiary teaching hospital
}

\author{
Jun $\mathrm{Li}^{1}$, Qiang Xu ${ }^{1}$, Haiyong Chen ${ }^{2,3}$, Lingling Tang ${ }^{4}$, Kai Yang ${ }^{5}$, Linrun Wang ${ }^{1}$, Xiaoyang Lu ${ }^{1}$, \\ Yuefeng Rao ${ }^{1}$
}

${ }^{1}$ Department of Clinical Pharmacy, the First Affiliated Hospital, Zhejiang University School of Medicine, Hangzhou, China; ${ }^{2}$ Department of Medical Administration, the First Affiliated Hospital, Zhejiang University School of Medicine, Hangzhou, China; ${ }^{3}$ Department of Surgery, Division of Hepatobiliary and Pancreatic Surgery, the First Affiliated Hospital, Zhejiang University School of Medicine, Hangzhou, China; ${ }^{4}$ Department of Hospital Infection Management, the First Affiliated Hospital, Zhejiang University School of Medicine, Hangzhou, China; ${ }^{5}$ Department of Urology Surgery, the First Affiliated Hospital, Zhejiang University School of Medicine, Hangzhou, China

Contributions: (I) Conception and design: J Li, X Lu, Y Rao; (II) Administrative support: L Wang, X Lu; (III) Provision of study materials or patients: L Tang, K Yang, X Lu; (IV) Collection and assembly of data: J Li, H Chen, L Tang, K Yang; (V) Data analysis and interpretation: J Li, Q Xu; (VI) Manuscript writing: All authors; (VII) Final approval of manuscript: All authors.

Correspondence to: Yuefeng Rao. Department of Pharmacy, the First Affiliated Hospital, Zhejiang University School of Medicine, Hangzhou 310003, China. Email: raoyf@zju.edu.cn.

Background: The spread of carbapenem-resistant Gram-negative bacteria poses a substantial threat to morbidity and mortality worldwide, which is mainly attributed to the overuse of carbapenem. This study aimed to evaluate the use of a quality control circle (QCC) in controlling the overuse of carbapenems and improving the state of carbapenem resistance at a Chinese tertiary teaching hospital.

Methods: A pharmacist-led multidisciplinary QCC project was carried out and the plan-do-check-act (PDCA) method was applied for 12 months. The data on carbapenem consumption, bacterial identification, and antibacterial susceptibility testing were collected to evaluate the effect of this project.

Results: The antibiotics use density (AUD) of carbapenems exhibited a decreasing trend over time $(\mathrm{P}<0.001)$, and the AUD of meropenem, imipenem, and biapenem decreased by $30.20 \%, 42.45 \%$, and $78.05 \%$ after the intervention, respectively. The total AUD of carbapenems decreased from 7.37 to 3.96, which included the decrease in the irrational use of carbapenems by 1.61 , accounting for $47.21 \%$ of the total. Moreover, the positive correlations were discovered between the resistance rate of carbapenem-resistant Klebsiella pneumonia (CRKP)/Acinetobacter baumannii (CRAB) and the AUD of carbapenems $(\mathrm{P}<0.05)$. The resistance rate of CRKP and CRAB decreased from $51.93 \%$ and $89.21 \%$ to $32.94 \%$ and $60.66 \%$, respectively, following QCC project implementation.

Conclusions: This is the first study to highlight the success of a multifaceted intervention QCC project and PDCA method, which led to a significant reduction in the AUD and resistance rate of carbapenems. QCC is a feasible and effective management tool for improving the quality of carbapenem use in medical institutions.

Keywords: Carbapenems; quality control circle (QCC); antibiotics use density (AUD); antimicrobial resistance; antibiotic consumption

Submitted Aug 30, 2021. Accepted for publication Oct 20, 2021.

doi: 10.21037/apm-21-2644

View this article at: https://dx.doi.org/10.21037/apm-21-2644 


\section{Introduction}

China is in the first-tier among the world's most frequent users of antibiotics (1). In the hospital setting, about $70 \%$ of inpatients and $20 \%$ of outpatients in China were prescribed antibiotics by doctors before 2011, which are both twice expectation of World Health Organization (WHO) for these rates (2). The overuse of antibiotics may not only adversely affect their therapeutic efficacy but also may substantially increase the cost of health care. Therefore, along with the WHO's appeal of "against drug resistance: no action today, no drugs tomorrow," the Chinese government in April 2011 officially launched an unprecedentedly strict protocol concerning the clinical use of antibiotic agents (3). Since then, the antibiotic use density (AUD) and use rate have both been significantly reduced. From 2011 to 2017, the use rate of antibiotics for outpatients decreased by about $50 \%$ (from $16.2 \%$ to $8.1 \%$ ), and AUD [defined daily dose (DDD) per 100 patient-days] decreased from 70.4 to 49.7 .

Under the remarkable success on the antimicrobial stewardship program (ASP), the consumption of many antibiotics decreased significantly. However, during the same period, the consumption of carbapenems in medical institutions in China also increased significantly $(3,4)$. The antibiotic consumption of different carbapenems increased by about $5.69 \%$ to $42.98 \%$ over the 6 years preceding 2017. Carbapenems are powerful broad-spectrum $\beta$-lactam antibiotics that are widely regarded by clinicians as "last-line" antibiotics, particularly for the management of critically ill patients and/or those with antimicrobialresistant Gram-negative infections. However, the irrational use and overuse of carbapenems has already led to the emergence of a large number of carbapenem-resistant bacteria strains $(5,6)$. According to the report of China Antimicrobial Resistance Surveillance System in 2018, the resistance rates of carbapenem antibiotics to carbapenemresistant Acinetobacter baumannii (CRAB), carbapenemresistant Pseudomonas aeruginosa (CRPA) and carbapenemresistant Klebsiella pneumoniae (CRKP) in China were 56.1\%, $19.3 \%$ and $10.1 \%$, respectively. Moreover, the WHO created a priority list of antibiotic-resistant bacteria to direct research and development of new and effective antibiotics in 2017 (7). In this list, CRAB, CRPA and CRKP were all included in the critical-priority bacteria list. Carbapenemresistant Gram-negative bacteria (CRGN) represent an escalating global public health threat (8). Therefore, how to effectively control the overuse of carbapenems and improve the state of carbapenem-resistance is an urgent problem that requires an immediate solution.

The quality control circle (QCC), as a process management and problem-solving technique, was first used in business management and company operations in Japan $(9,10)$. With the joint efforts of all members in an institution informed by the procedures of QCC, the institution can optimally leverage its advantages, cooperate with other related departments, simplify complex workflows, and ultimately resolve problems in work and management $(9,11)$. Since 2001, QCC was introduced to medical institutions in China in order to improve the quality of medical services by strengthening medical workers' awareness for discovering and solving medical problems, it is widely used in many aspects of improving medical quality. For example to reduce blood culture contamination rates for department of clinical laboratory (12), improve the intraocular lens power calculation for ophthalmology department (13), improve the hand hygiene compliance (14), reduce the incidence of catheter-associated urinary tract infection for department of nosocomial infection control (15), and so on. However, to our knowledge, no studies have been reported on QCC reducing carbapenem resistance. Therefore, in this study, a pharmacist-led QCC project was carried out at the first affiliated hospital of Zhejiang university (FAHZU), a top medical facility in China, with the aim of reducing the irrational use of carbapenems and improving the state of carbapenem resistance under QCC principles. We present the following article in accordance with the Materials Design Analysis Reporting (MDAR) checklist (available at https://dx.doi.org/10.21037/apm-21-2644).

\section{Methods}

\section{Establishment of improvement team}

The QCC project was launched based on the principles of QCC (16). The basic steps of the QCC project are outlined in Figure 1, with its implementation following the plan-do-check-act (PDCA) framework, a repetitive four-stage model for continuous improvement in quality management. According to the concept of multidisciplinary collaboration on antimicrobial stewardship, a QCC project team was established and enrolled members from different departments, including clinical pharmacy, medical administration, hospital infection management, and clinical microbiology laboratory. Some clinicians were also included in the team. All members met regularly for all stages of the project to provide suggestions and discuss project progress, 


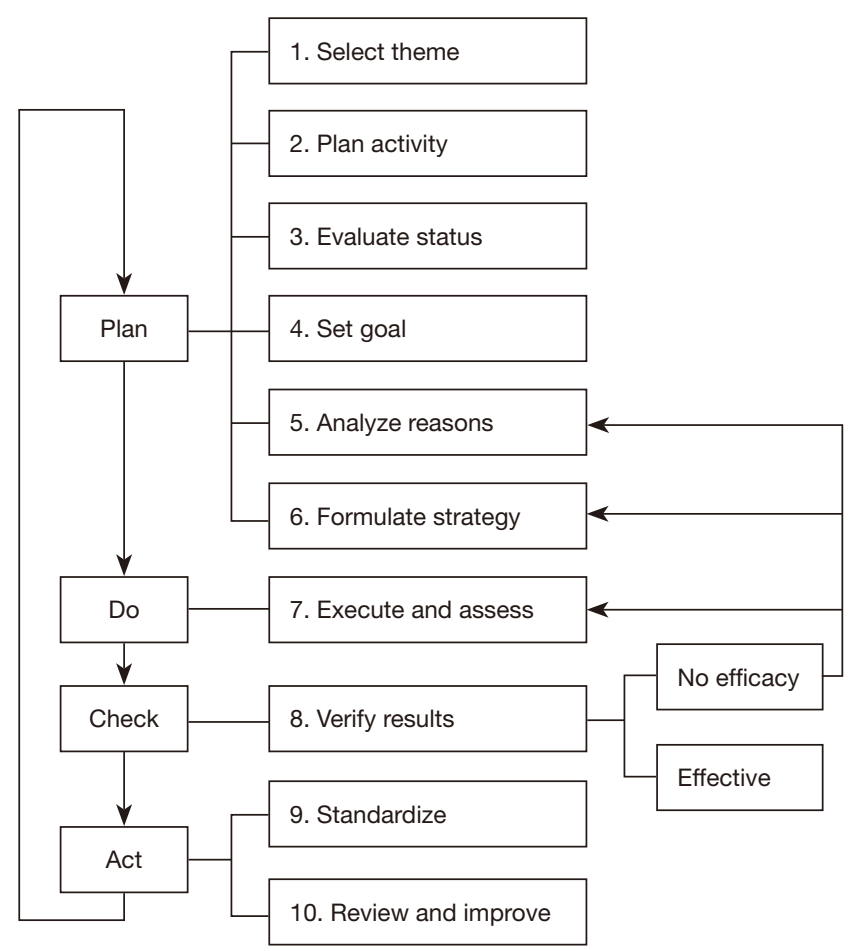

Figure 1 Protocol of the QCC in 10 steps and the PDCA workflow. QCC, quality control circle; PDCA, plan-do-check-act. aiming to work together from multiple dimensions to standardize the rational use of carbapenems in our hospital. The model of multidisciplinary collaboration could help to greatly improve the effectiveness of the QCC project.

\section{Baseline status evaluation and major reasons analysis}

In order to evaluate the baseline status of AUD and the rate of rational carbapenem usage in hospital, we retrospectively analyzed all the cases in 2017 in which carbapenems were used during hospitalization. After this, a fishbone analytical diagram method was adopted to analyze the potential reasons for the high AUD of carbapenem use in 4 aspects: human, equipment, environment, and management (Figure 2). Fishbone analysis is a method of brainstorming that identifies as many conceivable causes for a specific problem as possible (17). Following this, all QCC members voted to choose which of these causes were the most important and which were the critical issues that needed to be resolved in the QCC project.

\section{Formulation of strategies and interventions}

After identifying the most important reasons, all members

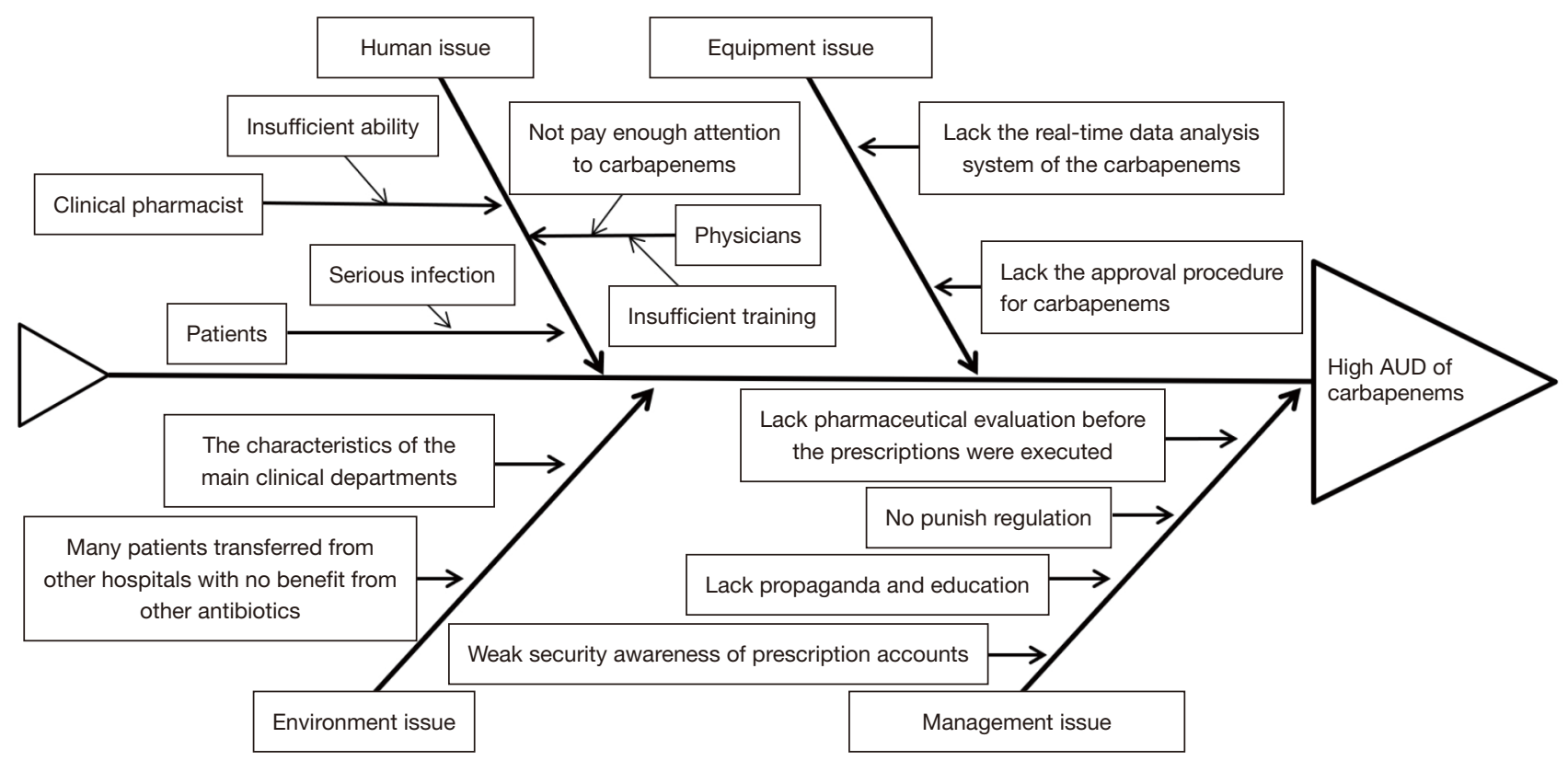

Figure 2 Fishbone analytical diagram. This fishbone diagram includes the main reasons identified by QCC members that lead to high AUD of carbapenems in medical institutions. QCC, quality control circle; AUD, antibiotic use density. 
of the QCC project discussed multiple possible strategies for each major problem in the form of a brainstorm. Subsequently, the strategies were graded by all members of the project based on a 5 -point system, considering from three aspects included feasibility, economy and circle ability (13). Those strategies that received a higher score for each major issue were selected. During this process, the necessary number of team meetings were held to optimize the strategies, and 4 intervention strategies were formulated for our QCC project as described below.

The first intervention was to carry out the pharmaceutical evaluation for the use of carbapenems with physicians before the prescriptions were executed. After an analysis of the state of carbapenem usage in our hospital, it was found that the rationality evaluation of carbapenem prescription was insufficient. The prescription audit at that time mainly focused on the administration frequency and dosage of carbapenems, as well as the liver and kidney function of patients. However, it was rare for pharmacists to evaluate the necessity of using carbapenems from the view of the patients' condition of infection. Moreover, the existing intervention was mainly carried out after the prescriptions' execution and could not prevent the execution of irrational clinical orders for carbapenems in time. Therefore, we designed a system for pharmaceutical evaluation and integrated it into Hospital Information System (HIS). This mainly consisted of conducting pharmaceutical evaluation for carbapenems before the prescriptions were administered at bedside and strengthening communication with physicians, in order to prevent irrational carbapenem use.

The second intervention involved further restricting the authority to prescribe carbapenems and strengthening the account security of the electronic medical order system within the medical institution. In general, it is relatively easy to obtain carbapenems for clinicians who have prescription authority in China. Moreover, for the convenience of work, clinicians without authority often prescribe carbapenems using the account of other clinicians on the same medical team who do have prescription authority. Therefore, a multisectoral approval procedure for carbapenems was established in order to improve the transparency of carbapenem usage and further strengthen the regulatory process through the use of a mobile phone APP. The approval process included the confirmation of the clinical department chief, the pharmacy department, and the medical administration department.

The third intervention was to strengthen the awareness and education of clinicians concerning the rational use of carbapenems and prescription evaluation. According to an analysis of baseline status, a major potential reason for overuse and misuse of carbapenems was that the clinicians, especially the surgeons in surgical wards, lacked the relevant knowledge and paid insufficient attention to the requirements for the rational use of carbapenems. Therefore, collaboration with the department of hospital infection management was initiated to carry out faceto-face communication and awareness-raising in clinical departments with serious irrational usage of carbapenems. This proved to be essential for strengthening the surgeons' awareness of the rational carbapenem usage. Moreover, financial penalties and revocation of prescription privileges based on a point-based prescription evaluation of irrational drug use were implemented.

The final intervention involved the design of the information system and the provision of real-time carbapenem usage data available to clinical department heads. During the QCC project, we received feedback from the heads of clinical departments. One issue that was frequently mentioned was the lack of real-time carbapenem usage data. Managers of clinical departments could not obtain carbapenem usage information from the department members in time and thus could not make management decisions using up-to-date data. Consequently, we established a real-time data acquisition function for the department heads based on the HIS. This could be used to analyze the carbapenem usage data for each department and each clinician within a department. This data acquisition function can provide data support for the department head and facilitate self-management of the clinical departments.

\section{Data collection and outcome measures}

During the implementation of the interventions, the data of bacterial identification and antibacterial susceptibility testing were provided by the hospital's clinical microbiology laboratory as described in our previous publication (18). The pathogens isolated from in-patients were identified by the VITEK 2 compact system (bioMérieux SA, Marcy-l'Étoile, France), and antibiotic susceptibility was determined by either the disc diffusion method or the VITEK 2 compact system. The results were then interpreted uniformly in accordance with the Clinical and Laboratory Standards Institute criteria. All positive clinical specimens, specifically those positive for $K$. pneumonia, A. baumannii, and $P$. aeruginosa, were included. To guarantee the reproducibility of testing methodologies, we used Enterococcus faecalis 
[American Type Culture Collection (ATCC) 29212], P . aeruginosa (ATCC 27853), and Escherichia coli (ATCC 25922) as reference strains.

The consumption data of carbapenems were obtained from the database of the pharmacy department. The AUD was calculated according to the following formula prescribed by the WHO. AUD of carbapenems = DDD of carbapenems per 100 patient-days. The DDD is the assumed average maintenance dose per day of the corresponding antibiotic used for its main indication in adults.

The study was conducted in accordance with the Declaration of Helsinki (as revised in 2013). No specific patients were involved in this study; thus, ethical approval and consent were waived.

\section{Statistical analysis}

Carbapenems consumption trends were analyzed by

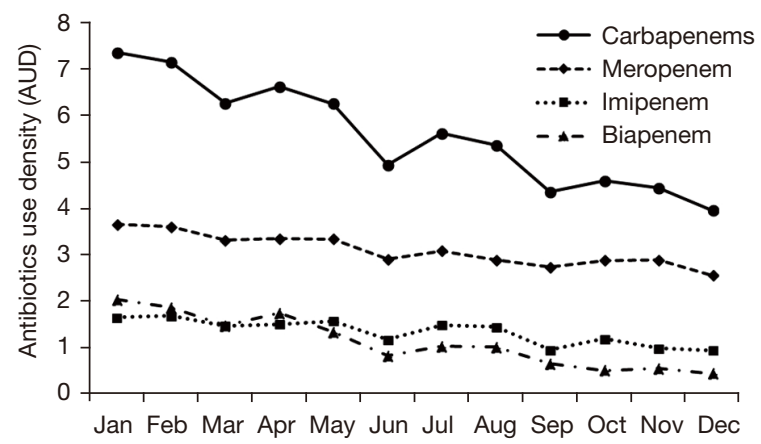

Figure 3 The monthly change trend of carbapenem consumption during the QCC project in 2018. Three carbapenems (meropenem, imipenem, and biapenem) were included. The AUD of carbapenems was defined as the daily dose per 100 patient-days. QCC, quality control circle; AUD, antibiotic use density. regression analysis, and Spearman correlation analysis was adopted to investigate the association between antibiotic consumption and the rate of carbapenem resistance. All the data were analyzed using SPSS version 22.0 (RRID: SCR_002865; IBM Corporation, Armonk, NY, USA). Throughout the work, $\mathrm{P}$ values less than 0.05 were considered to be statistically significant.

\section{Results}

\section{Change trends of carbapenem consumption during the QCC project}

From January 2018 to December 2018, the AUD of carbapenems during the QCC project were analyzed each month. The results demonstrated that the adopted intervention strategies gradually produced the expected effect on reducing the AUD of carbapenems as the QCC project progressed. As shown in Figure 3 and Table 1, the overall AUD of carbapenems decreased from 7.37 to 3.96 after 12 months of intervention and significantly decreased over time $(\mathrm{P}<0.001)$. Moreover, consistent with a previous study (3), meropenem was the most consumed among the 3 carbapenems in our hospital. The AUD of meropenem, imipenem, and biapenem decreased by $30.20 \%, 42.45 \%$, and $78.05 \%$ after the interventions, respectively $(\mathrm{P}<0.001)$. More interestingly, the reduction on the AUD of carbapenems could be mainly attributed to the control on the irrational usage of carbapenems. The AUD of irrational carbapenems usage decreased from 2.14 to 0.53 , and accounted for $47.21 \%$ of the total reduction of the carbapenem AUD.

\section{The correlations between the resistance rates of CRGN and carbapenems consumption}

Furthermore, the correlation between the resistance rates

Table 1 The trends in the consumption of carbapenems in 2018

\begin{tabular}{|c|c|c|c|c|c|}
\hline Item & AUD & Percentage of decrease & Trend & Slope $(\beta)$ & $\mathrm{P}$ \\
\hline Imipenem & $1.47(0.95-1.69)$ & $42.45 \%$ & Decreasing & -0.066 & $<0.001$ \\
\hline Biapenem & $1.03(0.45-2.05)$ & $78.05 \%$ & Decreasing & -0.148 & $<0.001$ \\
\hline Carbapenems & 5.49 (3.96-7.37) & $46.26 \%$ & Decreasing & -0.304 & $<0.001$ \\
\hline
\end{tabular}

The carbapenem consumption was defined as daily dose per 100 patient-days (AUD), and the values are presented as median (interquartile range). AUD, antibiotic use density. 


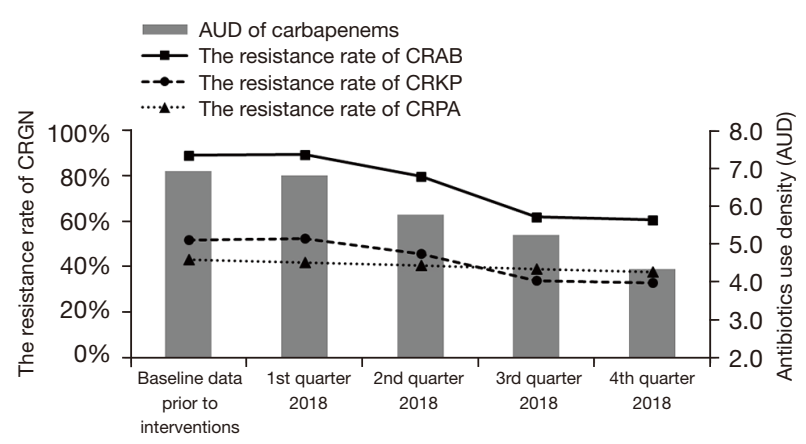

Figure 4 The trends and correlations between the resistance rates of carbapenem-resistant Gram-negative bacteria and the consumption of carbapenem during the QCC project. The AUD of carbapenems was defined as the daily dose per 100 patientdays. QCC, quality control circle; AUD, antibiotic use density; CRGN, carbapenem-resistant Gram-negative bacteria; CRKP, carbapenem-resistant Klebsiella pneumoniae; CRAB, carbapenemresistant Acinetobacter baumannii; CRPA, carbapenem-resistant Pseudomonas aeruginosa.

of CRGN and carbapenems consumption was analyzed. Positive correlations were discovered between the resistance rates of CRKP/CRAB and the AUD of carbapenems, with coefficients of 0.912 and 0.951 , respectively $(\mathrm{P}<0.05)$. The resistance rates of CRKP and CRAB decreased from $51.93 \%$ and $89.21 \%$ to $32.94 \%$ and $60.66 \%$, respectively, with the reduction of the carbapenem AUD after 12 months of intervention. However, no significant correlation was found between the resistance rate of CRPA and carbapenem consumption $(\mathrm{P}=0.617)$. The trend for the resistance rate of CRPA remained stable (Figure 4).

\section{The effect of QCC in optimizing the management of carbapenems}

In addition to the data-based results, this project has further improved the management process of carbapenems and optimized the information system settings in our hospital, which now include the regular carrying out of bedside pharmaceutical evaluation for carbapenem use before prescription and the establishment of a multisectoral approval procedure for carbapenems, among other initiatives. This project has also provided each clinical department the means for self-management concerning the use of carbapenems. The implementation of these institutional initiatives will better guarantee the success of this project.

\section{Discussion}

Antibiotic abuse has become a serious social issue that threatens public health on a global scale. The spread of antibiotic-resistant bacteria poses a substantial threat to morbidity and mortality worldwide (19). The global overuse and misuse of carbapenems has led to a further enhancement of bacterial resistance (5). Therefore, in 2017, National Health and Family Planning Commission of the People's Republic of China (NHFPC) launched a special scheme for the clinical application of carbapenems in order to further strengthen their management. Correspondingly, the FAHZU also implemented the following interventions for carbapenem management: (I) surveillance and regular proclamation of antibiotic resistance data and proportion of patients receiving antibiotic prescriptions; (II) according to the relevant requirements of government departments, limitations on the authority for the use of carbapenems based on the technical title of physician; and (III) the collaboration between physicians and pharmacists and the Infectious Disease Department in the FAHZU to improve the practice of health care workers. Due to these interventions, the clinical application of carbapenems in the FAHZU has been improved to some extent, but some shortcomings remain. For example, the susceptibility to carbapenems of $K$. pneumoniae has decreased significantly. In our previous study $(6,18)$, correlation analysis indicated that the increasing consumption of carbapenems may contribute to the burden of CRKP. Therefore, how to achieve continuous quality improvement is an urgent issue that needs to be solved.

QCC has proven to be a useful management technique for medical institutions. Therefore, in this study, we carried out a pharmacist-led QCC project with the aim of reducing the AUD of carbapenem and achieved noteworthy results. After an intervention of 12 months, the AUD of carbapenems decreased from 7.37 to 3.96 , and exhibited a significant decreasing trend over time. Our results also confirmed that proper management of antibiotics can decelerate or even reverse bacterial resistance. The resistance rates of CRKP and CRAB were significantly correlated with the AUD of carbapenems and decreased respectively with the reduction of the carbapenem consumption during this project. Although some medical institutions have carried out ASP, to our knowledge, this is the first report to apply QCC and PCDA management tools 
during the process of ASP, which provides specific methods for the efficient implement of ASP, including fishbone analytical diagram, brainstorming and so on.

Moreover, the promotion of QCC activities can not only obtain tangible results such as reduction of the AUD and resistance rate of carbapenems, but also produce intangible benefits (13). For example, the QCC model can better realize the team's capabilities and potential abilities. The members of project are willing to spend more time, energy, and creativity in contributing to the harmonious development of the team and its success. In addition, given that quality control activities involve many different departments, a sense of responsibility and coordination is promoted. After all, the model of solving problems by a single department is no longer the ideal method in modern society. In addition, QCC activities also exert a certain incentive effect on morale and work attitude. Generally, the more active QCC is, the better results we can achieve, from which every member can benefit. Although this preliminary study was only conducted in a single Chinese tertiary hospital, we hope our results can inspire further research into the role of QCC on antimicrobial management in other medical institutions.

Despite this study's success, several limitations should also be noted. (I) Baseline data analysis indicated that irrational usage of carbapenems is mainly attributed to the surgical wards. Therefore, in order to achieve a better clinical outcome, the intervention of this project could mainly focus on surgical wards, given the limitations of the circle method. (II) This was a 1-year pharmacistled QCC project for sustained improvement on the state of carbapenem resistance at a single tertiary hospital. Extending the implementation cycle of the project might offer a better evaluation and more authentically reflect the ability of the QCC project to sustain a reduction in carbapenem resistance. Therefore, further studies regarding the QCC method and the prolonged reduction of carbapenem consumption and resistance rates should be conducted using a longer time span and a greater number of medical institutions.

\section{Conclusions}

QCC is a feasible and effective management tool to control the abuse of carbapenems and reduce the rate of bacterial resistance to carbapenems. Moreover, multi-disciplinary collaboration involving the pharmaceutical sector is very important in antibiotic management. Pharmacists play an important role in the antimicrobial stewardship program.

\section{Acknowledgments}

Funding: This work was supported by the Zhejiang Provincial Natural Science Foundation Project (No. LY18H160017).

\section{Footnote}

Reporting Checklist: The authors have completed the MDAR checklist. Available at https://dx.doi.org/10.21037/apm-212644

Data Sharing Statement: Available at https://dx.doi. org/10.21037/apm-21-2644

Conflicts of Interest: All authors have completed the ICMJE uniform disclosure form (available at https://dx.doi. org/10.21037/apm-21-2644). The authors have no conflicts of interest to declare.

Ethical Statement: The authors are accountable for all aspects of the work in ensuring that questions related to the accuracy or integrity of any part of the work are appropriately investigated and resolved. The study was conducted in accordance with the Declaration of Helsinki (as revised in 2013). No specific patients were involved in this study; thus, ethical approval and consent were waived.

Open Access Statement: This is an Open Access article distributed in accordance with the Creative Commons Attribution-NonCommercial-NoDerivs 4.0 International License (CC BY-NC-ND 4.0), which permits the noncommercial replication and distribution of the article with the strict proviso that no changes or edits are made and the original work is properly cited (including links to both the formal publication through the relevant DOI and the license). See: https://creativecommons.org/licenses/by-nc-nd/4.0/.

\section{References}

1. Wang Z, Zhang H, Han J, et al. Deadly Sins of Antibiotic Abuse in China. Infect Control Hosp Epidemiol 2017;38:758-9.

2. Li Y, Xu J, Wang F, et al. Overprescribing in China, driven by financial incentives, results in very high use of antibiotics, injections, and corticosteroids. Health Aff 
(Millwood) 2012;31:1075-82.

3. Zhang D, Hu S, Sun J, et al. Antibiotic consumption versus the prevalence of carbapenem-resistant Gramnegative bacteria at a tertiary hospital in China from 2011 to 2017. J Infect Public Health 2019;12:195-9.

4. Qu X, Yin C, Sun X, et al. Consumption of antibiotics in Chinese public general tertiary hospitals (2011-2014): Trends, pattern changes and regional differences. PLoS One 2018;13:e0196668.

5. Perez F, Bonomo RA. Evidence to improve the treatment of infections caused by carbapenem-resistant Gramnegative bacteria. Lancet Infect Dis 2018;18:358-60.

6. Yang P, Chen Y, Jiang S, et al. Association between antibiotic consumption and the rate of carbapenemresistant Gram-negative bacteria from China based on 153 tertiary hospitals data in 2014. Antimicrob Resist Infect Control 2018;7:137.

7. Tacconelli E, Carrara E, Savoldi A, et al. Discovery, research, and development of new antibiotics: the WHO priority list of antibiotic-resistant bacteria and tuberculosis. Lancet Infect Dis 2018;18:318-27.

8. Laxminarayan R, Matsoso P, Pant S, et al. Access to effective antimicrobials: a worldwide challenge. Lancet 2016;387:168-75.

9. Matsuda K. Definition of the QC (quality control) circle activities. Kango Tenbo 1983;8:51-7.

10. Munchus G 3rd. Employer-employee based quality circles in Japan: human resource policy implications for American firms. Acad Manage Rev 1983;8:255-61.

11. Krause G, Benzler J, Reiprich G, et al. Improvement of a national public health surveillance system through use of a

Cite this article as: $\mathrm{Li} \mathrm{J,} \mathrm{Xu} \mathrm{Q,} \mathrm{Chen} \mathrm{H,} \mathrm{Tang} \mathrm{L,} \mathrm{Yang} \mathrm{K,}$ Wang L, Lu X, Rao Y. Pharmacist-led quality control circle in sustained reduction of carbapenem-resistance at a Chinese tertiary teaching hospital. Ann Palliat Med 2021;10(11):1155811565. doi: 10.21037/apm-21-2644 quality circle. Euro Surveill 2006;11:246-8.

12. Yan YZ, Yang HZ, Zhao JX, et al. A Quality Control Circle Process to Reduce Blood Culture Contamination Rates. Infect Control Hosp Epidemiol 2019;40:119-20.

13. Lin L, Chang P, Xie J, et al. Sustained accuracy improvement in intraocular lens power calculation with the application of quality control circle. Sci Rep 2017;7:14852.

14. Chen P, Yuan T, Sun Q, et al. Role of quality control circle in sustained improvement of hand hygiene compliance: an observational study in a stomatology hospital in Shandong, China. Antimicrob Resist Infect Control 2016;5:54.

15. Zhong X, Xiao L, Wang D, et al. Impact of a quality control circle on the incidence of catheter-associated urinary tract infection: An interrupted time series analysis. Am J Infect Control 2020;48:1184-8.

16. Wang LR, Wang Y, Lou Y, et al. The role of quality control circles in sustained improvement of medical quality. Springerplus 2013;2:141.

17. Gold J, Reyes-Gastelum D, Turner J, et al. A quality improvement study using fishbone analysis and an electronic medical records intervention to improve care for children with asthma. Am J Med Qual 2014;29:70-7.

18. Wang R, Yang Q, Zhang S, et al. Trends and correlation of antibiotic susceptibility and antibiotic consumption at a large teaching hospital in China (2007-2016): a surveillance study. Ther Clin Risk Manag 2019;15:1019-27.

19. Burnham CD, Leeds J, Nordmann P, et al. Diagnosing antimicrobial resistance. Nat Rev Microbiol 2017;15:697-703.

(English Language Editor: J. Gray) 